\title{
Multiwavelength studies of WR 21a and its surroundings
}

\author{
P. Benaglia ${ }^{1,2, \star}$, G. E. Romero ${ }^{1,2, \star}$, B. Koribalski ${ }^{3}$, and A. M. T. Pollock ${ }^{4}$ \\ 1 Instituto Argentino de Radioastronomía, C.C.5, (1894) Villa Elisa, Buenos Aires, Argentina \\ e-mail: pbenaglia@fcaglp.unlp.edu.ar \\ 2 Facultad de Cs. Astronómicas y Geofísicas, UNLP, Paseo del Bosque s/n, (1900) La Plata, Argentina \\ 3 Australia Telescope National Facility, CSIRO, PO Box 76, Epping, NSW 1710, Australia \\ 4 XMM-Newton Science Operations Centre, European Space Astronomy Centre, Apartado 50727, 28080 Madrid, Spain
}

Received 27 December 2004 / Accepted 6 June 2005

\begin{abstract}
We present results of high-resolution radio continuum observations towards the binary star WR 21a (Wack 2134) obtained with the Australia Telescope Compact Array (ATCA ${ }^{\star \star}$ ) at 4.8 and $8.64 \mathrm{GHz}$. We detected the system at $4.8 \mathrm{GHz}$ $(6 \mathrm{~cm})$ with a flux density of $0.25 \pm 0.06 \mathrm{mJy}$ and set an upper limit of $0.3 \mathrm{mJy}$ at $8.64 \mathrm{GHz}(3 \mathrm{~cm})$. The derived spectral index of $\alpha<0.3\left(S_{v} \propto v^{\alpha}\right)$ suggests the presence of non-thermal emission, probably originating in a colliding-wind region. A second, unrelated radio source was detected $\sim 10^{\prime \prime}$ north of WR 21a at (RA, Dec) $)_{\mathrm{J} 2000}=\left(10^{\mathrm{h}} 25^{\mathrm{m}} 56^{\mathrm{s}} .49,-57^{\circ} 48^{\prime} 34.4^{\prime \prime}\right)$, with flux densities of 0.36 and $0.55 \mathrm{mJy}$ at 4.8 and $8.64 \mathrm{GHz}$, respectively, resulting in $\alpha=0.72$. H I observations in the area are dominated by absorption against the prominent H II region RCW 49. Analysis of a complete set of archived X-ray observations of WR 21a confirms its strong variability but throws into doubt previous suggestions by Reig (1999) of a period of years for the system. Finally, we comment on the association with the nearby EGRET source 3EG J1027-5817.
\end{abstract}

Key words. stars: early-type - stars: individual: WR 21a - stars: winds, outflows - radio continuum: stars - ISM: bubbles gamma-rays: observations

\section{Introduction}

Massive O and Wolf-Rayet (WR) stars lose large amounts of mass through dense, energetic winds. These winds can interact with the surrounding interstellar medium (ISM) to create cavities in the HI distribution (e.g. Benaglia \& Cappa 1999) or produce strong shock fronts that, in principle, can accelerate charged particles up to relativistic energies (e.g. Völk \& Forman 1982). In fact, non-thermal radio emission has been detected in a number of early-type stars, indicating the presence of populations of locally accelerated electrons (e.g. Chapman et al. 1999; Benaglia et al. 2001b; Benaglia \& Koribalski 2004).

Particle acceleration mediated by strong shocks could be taking place in a number of different regions of a massive stellar system such as the outer boundary of the interaction between the wind and the ISM (e.g. Cassé \& Paul 1980), the base of the wind, where line-driven instabilities are thought to drive strong shocks (e.g. White 1985), or, in massive binary systems, the colliding-wind region (CWR, e.g. Eichler \& Usov 1993). In addition to the expected non-thermal radio emission from relativistic electrons, high-energy radiation could be produced through, for example, inverse-Compton scattering of UV stellar photons or hadronic interactions from shock-accelerated ions

* Member of Carrera del Investigador, CONICET.

$\star \star$ The Australia Telescope is funded by the Commonwealth of Australia for operation as a National Facility managed by CSIRO. and ambient material (e.g. Pollock 1987; White \& Chen 1992; Romero et al. 1999; Benaglia et al. 2001a; Mücke \& Pohl 2002; Benaglia \& Romero 2003).

Only a small number of non-thermal radio WR binaries have been identified so far (see for instance Dougherty et al. 2003) and, even then, the connection between the radio and X-ray properties of these systems is far from clear. The archetype system WR 140 is bright in both regimes with a welldefined radio light curve that shares the 7.94-year period of the optical radial-velocity orbit. The X-ray light curve is not so well determined but shows strong variability consistent with the same period. WR 140, however, is the exception. The radio brightest star, WR 146, is a relatively faint X-ray source whereas WR 147 seems to be intermediate in both regimes. Sharing some characteristics with these systems, the star WR 21a $\left[(\alpha, \delta)_{\mathrm{J} 2000}=10^{\mathrm{h}} 25^{\mathrm{m}} 56^{\mathrm{s}} 49,-57^{\circ} 48^{\prime} 44.4^{\prime \prime},(l, \quad b)=\right.$ $\left.284^{\circ} .52,-0.24\right]$, is particularly interesting for various reasons. In the past, it coincided within the fairly large positional uncertainties with the COS B source 2CG 284-00 (Goldwurm et al. 1987). More recently, the unidentified EGRET gamma-ray source 3EG J1027-5817 was detected southwest of WR 21a (Hartman et al. 1999), being the star 30 arcmin apart from the center of the gamma-ray source. The $95 \%$ probability radius of the EGRET source is about $0.3^{\circ}$.

Caraveo et al. (1989) established that WR 21a is associated with the X-ray source 1E 1024.0-5732, and reported X-ray 
pulses. Its X-ray emission could be explained if the star is part of a binary system with either a compact companion forming a high-mass X-ray binary (Caraveo et al. 1989) or another massive, early-type star, with a CWR (Reig 1999). According to its stellar spectrum, it was early classified as an O5 star (Caraveo et al. 1989), and as a likely binary (WN5-6+O3f), at a maximum distance of $3 \mathrm{kpc}$ (Reig 1999).

With the current investigation we aim at determining the radio properties of the star and its surroundings. Specifically, using interferometric observations at 3 and $6 \mathrm{~cm}$, we looked for non-thermal radiation that might be interpreted as evidence for sites where electrons are being accelerated in colliding winds or terminal shocks. By means of low-resolution $21 \mathrm{~cm}$-line observations, we have also studied the distribution of neutral hydrogen around the star. The detection of non-thermal emission coincident with the stellar position would help in the identification of the system components. Analysis of all available X-ray data of WR 21a reveals a variability history that sheds fresh light on the nature of the binary. Finally, we discuss the possibility of a physical link between the stellar system and the nearby EGRET source, which can help to unveil the nature of the latter.

The contents of the paper are as follows: Sect. 2 reviews the main sources detected in this region of the sky that are relevant for the present study. Section 3 describes the observations carried out and the data reduction; Sect. 4 outlines the new observational results, whereas Sect. 5 presents the corresponding analysis. In Sect. 6 we discuss the X-ray data. Section 7 contains a comment on the possibility of a physical association between the star and the EGRET source 3EG J1027-5817. Section 8 closes with the summary.

\section{WR 21a and its surroundings}

The region of the Galactic plane towards $(l, b)=\left(284^{\circ}, 0^{\circ}\right)$ has been widely observed, from radio to gamma rays. The optical images of Rodgers et al. (1960) revealed various H II complexes, of which RCW 49 is the most prominent. The COS B satellite discovered the unidentified $\gamma$-ray source 2CG 284-00 (Bignami \& Hermsen 1983; Caraveo 1983). The zone was then investigated by means of Einstein X-ray measurements in which were detected emission from RCW 49 and a point source named 1E 1024.0-5732 (Goldwurm et al. 1987). The point source was tentatively linked to WR 21a (Hertz \& Grindlay 1984), an emission-line star with $m_{\mathrm{V}}=12.8$ (Wackerling 1969), also called Th35-42 and Wack 2134.

Caraveo et al. (1989) gathered enough evidence definitely to associate the X-ray source with the star. They took optical stellar spectra with the 3.6-m telescope at La Silla, classified the star as O5, set an upper limit for the stellar distance of $3 \mathrm{kpc}$, and studied the Einstein X-ray emission, reporting an X-ray periodicity of $\sim 60 \mathrm{~ms}$. They explained the X-ray behaviour as a binary with a compact companion, likely an accreting neutron star (NS). Dieters et al. (1990) looked for optical pulsations with the Tasmania 1-m telescope. They set an upper limit around 19.7 mag for any optical pulsation.

Mereghetti et al. (1994) observed the region with the ROSAT PSPC instrument, finding no pulsations, and obtained
Table 1. Adopted stellar parameters for the binary system WR 21a.

\begin{tabular}{lrrl}
\hline \hline Variable & $\mathrm{WR}$ & $\mathrm{OB}$ & Unit \\
\hline Spectral Class. & $\mathrm{WN}^{a}$ & $\mathrm{O} 3(\mathrm{I}) \mathrm{f}^{b}$ & \\
$\log \left(L / L_{\odot}\right)$ & $5.2^{c}$ & $6.274^{d}$ & \\
$T_{\text {eff }}$ & $45000^{c}$ & $50680^{d}$ & $\mathrm{~K}$ \\
$M_{*}$ & $12^{c}$ & $65^{d}$ & $M_{\odot}$ \\
$\mu$ & $4^{c}$ & $1.5^{c}$ & \\
$v_{\infty}$ & $2000^{c}$ & $3150^{c}$ & $\mathrm{~km} \mathrm{~s}^{-1}$ \\
$\dot{M}$ expected & $3.0 \times 10^{-5 c}$ & $1.4 \times 10^{-5 c}$ & $M_{\odot} \mathrm{yr}^{-1}$ \\
distance $d$ & $3^{c}$ & $3^{c}$ & $\mathrm{kpc}$ \\
\hline
\end{tabular}

${ }^{a}$ van der Hucht (2001); ${ }^{b}$ Reig (1999); ${ }^{c}$ see text.

${ }^{d}$ Vacca et al. (1996).

a further spectrum with the CTIO $1.6 \mathrm{~m}$ telescope, revising the spectral classification to WN6. Based on the small equivalent widths of the emission lines, they suggested that the object could be a binary with an $\mathrm{O}$ star, rather than a compact companion, in which case the X-rays could come from colliding winds, in common with other Wolf-Rayet binary systems.

In 1999, Reig presented RXTE data and a new spectrum taken with the $1.9 \mathrm{~m}$ telescope at SAAO. He stated that the lack of pulsations and the relatively soft and low X-ray emission seem to exclude the presence of a NS as responsible for the observed X-rays. Claiming that the spectrum shows features of both WR and O stars, he suggested that the system is formed by a WN6 and a possibly supergiant O3 companion, favoring the hypothesis of the colliding-wind binary (CWB). Roberts et al. (2001) observed the region towards WR 21a with ASCA, and interpreted the hard X-ray emission detected as produced by shocks from colliding winds in the stellar system. Very recently, Niemela et al.'s (2005) optical radial measurements have finally confirmed that the system is formed by two massive stars.

The distance $d$ to WR 21a is not well established. We shall adopt $d=3 \mathrm{kpc}$ throughout this paper, a value that seems to be consistent, as we will see, with all current observations.

Table 1 lists the adopted parameters that make up the Wack 2134/WR 21a binary system. Very few of the properties have so far been measured, so we have to assume parameters from similar stars or theoretical predictions. The stellar luminosity, effective temperature, and stellar mass of the WR component were estimated as an average of the same variables given by Hamann et al. (1995) for WN6 stars. The WR mean molecular weight of the ions $\mu$ was assumed the same as in Leitherer et al. (1997) for a WN6 star. The WR wind terminal velocity is taken as a mean value between data listed by Hamman et al. (1995) and by Prinja et al. (1990) for WN6 stars. The WR predicted mass loss rate $(\dot{M})$ is taken as the lowest value tabulated for WN6 stars by Nugis \& Lamers (2000), in their compilation of observable $\dot{M}$, and it can be considered a lower limit. The O3 (I) mass was taken as the spectroscopic mass from the tables of Vacca et al. (1996), and its terminal velocity from the averaged values listed by Prinja et al. (1990). We assumed $\mu=1.5$ for the $\mathrm{O}$ star because of its evolved stage. A predicted mass loss rate was estimated using the recipe derived 
by Vink et al. (2000) (astro.ic.ac.uk/ jvink/). The expected (WR+Of) mass-loss rate would imply a 6 -cm flux density of $0.24 \mathrm{mJy}$ at $3 \mathrm{kpc}$, if thermal emission from ionized winds in both stars is assumed.

Close to WR 21a there are two interesting sources: the HII region RCW 49, and the gamma-ray source 3EG J1027-5817. They are discussed below.

\subsection{The $\mathrm{H}$ II region $\mathrm{RCW} 49$}

RCW 49 is a southern HII region, located at $(l, b)=$ $\left(284.3^{\circ},-0.3^{\circ}\right)$, and extended over an area of $\sim 90^{\prime} \times 70^{\prime}$. Values for its distance range between 2.3 and $7.9 \mathrm{kpc}$ (Manchester et al. 1970; Moffat et al. 1991; Brand \& Blitz 1993, etc.). H I spectra towards RCW 49 taken by Goss et al. (1972) show prominent $\mathrm{HI}$ absorption from about -20 to $+5 \mathrm{~km} \mathrm{~s}^{-1}$ (see also Figs. 2 and 3). Whiteoak \& Uchida (1997) have imaged RCW 49 at radio continuum with MOST at $0.843 \mathrm{GHz}$, and the central region using ATCA at 1.38 and $2.38 \mathrm{GHz}$, attaining an angular resolution of 7 arcsec. They found two shells, and ascribe the formation of the northern one to the Westerlund 2 cluster containing the binary star WR 20a, and the southern one to the star WR 20b (see their Fig. 2d). Recent infrared images of RCW 49 obtained with the Spitzer Space Telescope (Churchwell et al. 2004) show the intricate filamentary structure of the nebula in the inner 5 arcmin shaped by stellar winds and radiation.

\subsection{The gamma-ray source 3EG J1027-5817}

After the analysis of the EGRET data, Hartman et al. (1999) were the first to point at the proximity between the gamma-ray source 3EG J1027-5817 and the X-ray source 1E 1024.0-5732, associated with WR 21a (see Fig. 3). The averaged measured flux at $E>100 \mathrm{MeV}$ is $65.9 \pm 0.70 \times$ $10^{-8}$ photons $\mathrm{cm}^{-2} \mathrm{~s}^{-1}$. The source is constant within errors on timescales of months (variability index $I=1.6$, see Torres et al. 2001) and with a photon spectral index $\Gamma=1.94 \pm 0.09$ $\left(\mathrm{d} N / \mathrm{d} E \propto E^{-\Gamma}\right)$. The more recent variability analysis by Nolan et al. (2003), who calculated a likelihood function for the flux of each source in each observation, also suggests that this source is not variable on short, monthly timescales.

\section{Observations and data reduction}

In order to search for non-thermal radio emission in the direction of WR 21a we have carried out interferometric radio continuum observations at high angular resolution $\left(1^{\prime \prime}-2^{\prime \prime}\right)$. These were complemented with single-dish $\mathrm{H}$ I-line observations to map the distribution of neutral material in the neighbourhood and study its kinematic behaviour.

\subsection{Radio continuum observations}

Radio continuum data were obtained in September 2001 with the Australia Telescope Compact Array in the 6D array, observing simultaneously at 3 and $6 \mathrm{~cm}$, or 8.64 and $4.8 \mathrm{GHz}$, respectively. The total bandwidth used was $128 \mathrm{MHz}$. The primary calibrator was PKS 1934-638, with flux densities of 5.83 and $2.84 \mathrm{Jy}$, at 4.8 and $8.64 \mathrm{GHz}$. WR 21a was tracked $12 \mathrm{~h}$ - full synthesis - to gain maximum $u v$ coverage, interleaving with observations of the phase calibrator 1039-47. The theoretical rms noise after $\sim 9 \mathrm{~h}$ on source is $0.03 \mathrm{mJy}$ at both frequencies, taking all baselines into account.

The data were reduced and analyzed with MIRIAD routines. The images built using "robust" weighting showed the best signal to noise ratio, and minimized sidelobes. The diffuse emission from extended sources was removed by taking out the visibilities corresponding to the shortest baselines. The resulting beams were $0.83^{\prime \prime} \times 0.70^{\prime \prime}$ at $3 \mathrm{~cm}$, and $1.73^{\prime \prime} \times 1.49^{\prime \prime}$ at $6 \mathrm{~cm}$. The rms noise of the final maps is $0.1 \mathrm{mJy}^{\text {beam }}{ }^{-1}$ at $3 \mathrm{~cm}$ and $0.06 \mathrm{mJy}$ beam $^{-1}$ at $6 \mathrm{~cm}$. The difference between the theoretical and the observed rms noise is mainly due to flagging of bad data - specially at $3 \mathrm{~cm}$ - as well as short baselines contributing with confusing emission from extended sources both in and outside the main beam.

The observations were set to optimize the detection of point-like features. Maps at two frequencies would allow the determination of spectral indices.

\subsection{HI-line observations}

The $21 \mathrm{~cm}$-line data were obtained with a $30 \mathrm{~m}$-single dish radiotelescope at the Instituto Argentino de Radioastronomía (IAR, Villa Elisa, Argentina) in June 2000. The observations were done in total power mode, covering a total field of $5^{\circ} \times 5^{\circ}$, in Galactic coordinates with a cell size of $15^{\prime} \times 15^{\prime}$. The $H P B W$ at $1420 \mathrm{MHz}$ is $30^{\prime}$. The receiver's system temperature was $\approx 35 \mathrm{~K}$. The velocity coverage was $(-450,+450) \mathrm{km} \mathrm{s}^{-1}$; the 1008 channel autocorrelator allows a maximum velocity resolution of $1.05 \mathrm{~km} \mathrm{~s}^{-1}$. The rms noise of the brightnesstemperature $\left(T_{\mathrm{B}}\right)$ of a single spectral point is $\sim 0.1 \mathrm{~K}$. The $T_{\mathrm{B}}$ scale was calibrated with the standard region S9 (Morras \& Cappa 1995). A series of $(l, b)-T_{\mathrm{B}}$ maps were built every $1.05 \mathrm{~km} \mathrm{~s}^{-1}$ to proceed with the analytical stage.

\section{Results}

\subsection{ATCA radio continuum data}

The ATCA images at 3 and $6 \mathrm{~cm}$ are shown in Fig. 1. A point source positionally coincident with WR 21 a is visible at $6 \mathrm{~cm}$, at $S / N>4$. A flux density $S_{6 \mathrm{~cm}}=0.25 \mathrm{mJy}$ was derived using IMFIT after a gaussian fit (see Table 2). The rms noise in the $3 \mathrm{~cm}$ image is $0.1 \mathrm{mJy}$ beam ${ }^{-1}$. No radio source is detected at the stellar position above $3 \mathrm{rms}$. This non-detection imposes an upper limit for the spectral index of $\alpha<0.3\left(S \propto v^{\alpha}\right)$. The deviation from purely thermal emission, characterized by $\alpha=$ 0.6-0.8, indicates the presence of a non-thermal contribution.

In a similar way as in Benaglia \& Koribalski (2004), we derive a mass loss rate of WR 21a, from the flux density at $6 \mathrm{~cm}$. In a first approximation, the mean number of electrons per ion $(\gamma)$, and the rms ionic charge $(Z)$ were taken equal to unity. The gas temperature is computed from the stellar effective temperature (see Table 1) as $0.4 T_{\text {eff. The }}$ Gaunt factor results in 5.6. At the adopted distance of $3 \mathrm{kpc}$, 

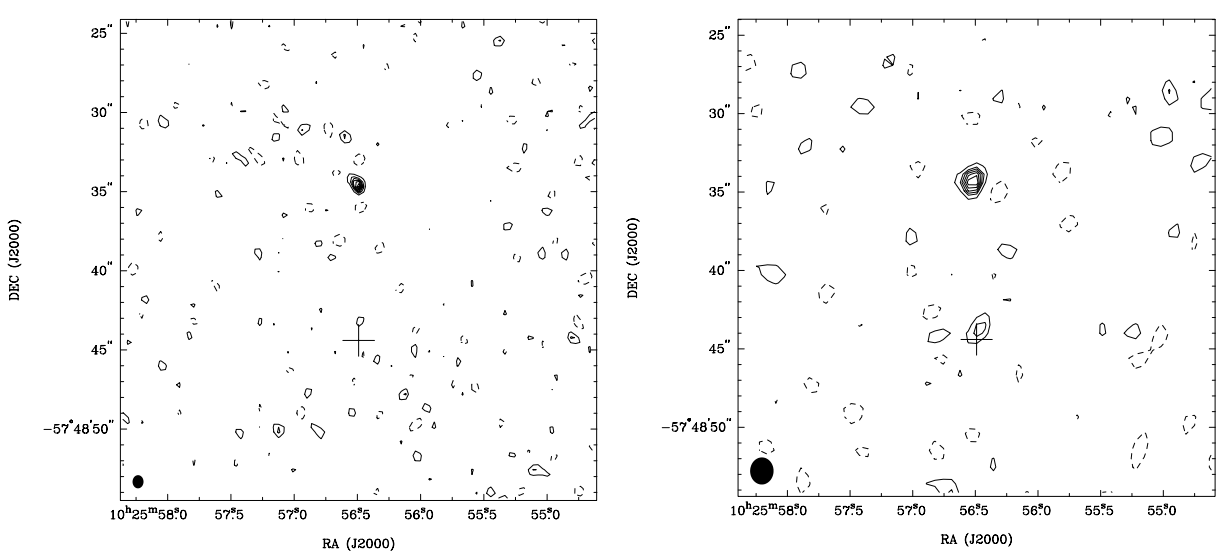

Fig. 1. Left panel: ATCA-3 cm radio continuum image towards WR 21a. The optical position of the star is marked with a cross. The contour levels are $-0.20,0.20(2 \sigma), 0.30,0.35,0.40,0.45,0.50$, and $0.55 \mathrm{mJy}_{\text {beam }}{ }^{-1}$. The synthesized beam $\left(0.83^{\prime \prime} \times 0.70^{\prime \prime}\right)$ is displayed at the bottom left corner. Right panel: ATCA-6 cm radio continuum image towards WR 21a. The contour levels are $-0.12,0.12(2 \sigma), 0.18,0.24,0.30$, and $0.33 \mathrm{mJy}_{\text {beam }}{ }^{-1}$. The synthesized beam $\left(1.73^{\prime \prime} \times 1.49^{\prime \prime}\right)$ is displayed at the bottom left corner.

Table 2. Radio continuum results.

\begin{tabular}{lrccc}
\hline \hline Object & $\begin{array}{r}S_{3 \mathrm{~cm}} \\
(\mathrm{mJy})\end{array}$ & $\begin{array}{c}S_{6 \mathrm{~cm}} \\
(\mathrm{mJy})\end{array}$ & $\begin{array}{c}\text { RA, Dec (J2000) } \\
(\mathrm{hms}, \mathrm{dms})\end{array}$ & $\alpha$ \\
\hline WR 21a & $<0.3$ & $0.25 \pm 0.06$ & $10: 25: 56.49-57: 48: 43.3$ & $<0.3$ \\
& & & & \\
S2 & $0.55 \pm 0.10$ & $0.36 \pm 0.06$ & $10: 25: 56.49-57: 48: 34.4$ & 0.72 \\
\hline
\end{tabular}

this would imply a radio-derived mass loss rate for the WR star of $\dot{M}=f \times 4.8 \times 10^{-5} M_{\odot} \mathrm{yr}^{-1}$, where $f$ is the fraction of thermal to total radio emission. From the relation between the flux density values measured with ATCA at 3 and $6 \mathrm{~cm}$ we know that at $6 \mathrm{~cm}$ there is a non-thermal contribution to the emission. Thus, in deriving a mass loss rate from the flux density at $6 \mathrm{~cm}$, the result with $f=1$ is an upper limit.

A second source, called S2, is detected 10" away from WR 21a, at both frequencies. The measured fluxes are $0.55 \mathrm{mJy}$ at $3 \mathrm{~cm}$, and $0.36 \mathrm{mJy}$ at $6 \mathrm{~cm}$. The corresponding spectral index of $\alpha_{\mathrm{S} 2}=+0.72$ points to an ultracompact $\mathrm{H}$ II region or the thermal wind of another, as yet unidentified, early-type star on the field of WR 21a. Table 2 lists the position and flux density of the two sources detected.

\subsection{Large-scale $H$ I-line data}

Studies of neutral hydrogen were performed to look for evidences of gas features such as shells, filaments, bubbles, etc., that could be physically related to the object under the present study. The H I gas kinematics in the line-of-sight to a Galactic source can be used to constrain its distance (e.g. Koribalski et al. 1995) using the Galactic rotation curve (e.g. Fich et al. 1989) and the Galactic velocity field (Brand \& Blitz 1993). Here we searched for the signatures of an interstellar H I bubble, created by the action of the stellar winds of WR 21a. Because of the large angular size of the IAR telescope beam $(H P B W=30$ arcmin; see Figs. 2 and 3$)$ and the proximity of WR 21a to the extended H II region RCW 49, which has a total radio continuum flux of $\sim 210 \mathrm{Jy}$ at $843 \mathrm{MHz}$ (Whiteoak \& Uchida 1997), H I spectra in this direction are completely dominated by H I absorption against RCW 49 (Goss et al. 1972; McClure-Griffiths et al. 2001). We also investigated the high-resolution (130 arcsec) H I data cubes from the Southern Galactic Plane Survey (SGPS; McClure-Griffiths et al. 2001) in the region of RCW 49 and WR 21a. Unfortunately, the region around RCW 49, including the $\mathrm{HI}$ line emission at the position of WR 21a, suffers from artifacts caused by the strong radio continuum emission from RCW49 and potential H I structures associated with WR 21a cannot be distinguished.

Figure 2 displays the $\mathrm{H}$ I brightness temperature maps each $4 \mathrm{~km} \mathrm{~s}^{-1}$ built from the IAR data.

If we were able to see an HI bubble around WR 21a, its size would give us some information about the energetics of the stellar wind and its velocity would give us an estimate of its kinematic distance using the Galactic rotation curve and velocity field. These issues are explored in the following section.

\section{Analysis of the line features}

The derivation of gas kinematic distances is difficult for this particular region of the Galactic plane. The line of sight goes tangential to the Carina arm at this Galactic longitude, and velocity crowding becomes very important. Brand \& Blitz (1993) showed that the measured velocities deviate strongly from Galactic rotation: gas in the velocity range from -20 to $-10 \mathrm{~km} \mathrm{~s}^{-1}$ can be located at distances between 2 and $6 \mathrm{kpc}$ for $l \sim 285^{\circ}$, where both WR 21a and RCW 49 are likely to be located.

By means of CO observations, Grabelsky et al. (1987) studied molecular gas associated with the Carina arm. They interpreted the velocity-longitude behaviour of the gas in terms of material at different heliocentric distances (see their Fig. 5), 


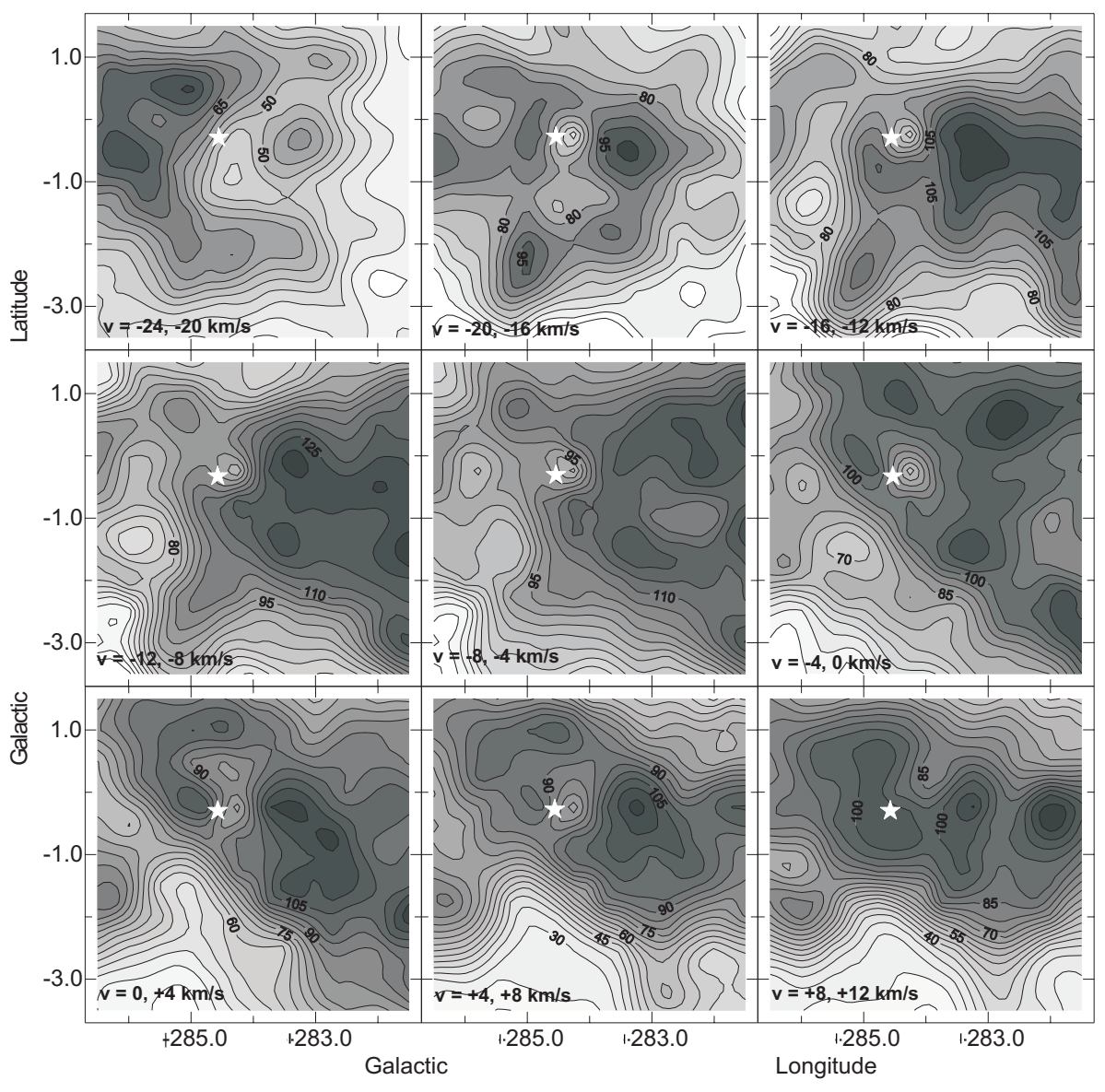

Fig. 2. H I-brightness temperature distribution of the Galactic emission as measured with the IAR telescope $(H P B W=30$ arcmin) over a velocity range from -24 to $+12 \mathrm{~km} \mathrm{~s}^{-1}$, in steps of $4 \mathrm{~km} \mathrm{~s}^{-1}$. The position of WR 21a is marked with a white star. The contour levels indicate H I brightness temperatures in steps of 5 Kelvin.

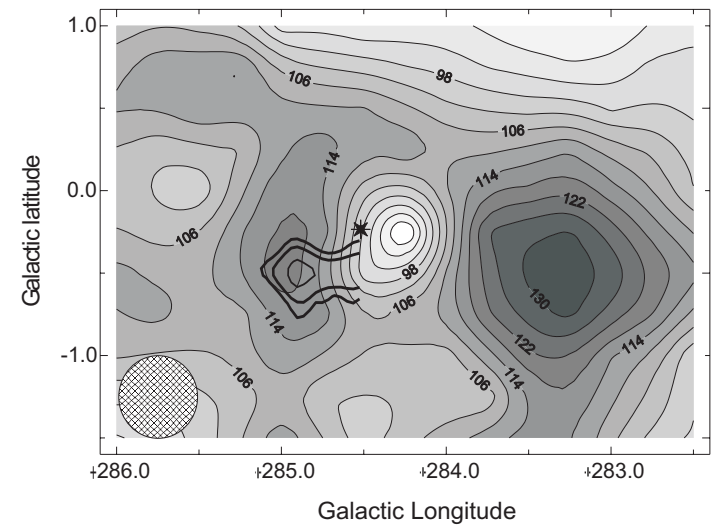

Fig. 3. Neutral-hydrogen column density integrated over velocities from -21 to $-14 \mathrm{~km} \mathrm{~s}^{-1}$. The contour levels indicate HI brightness temperatures in steps of 4 Kelvin. The IAR telescope beam is displayed in the bottom left corner. The position of WR 21a is marked with a black star, and the black contours represent the 99 , 95 and $50 \%$ probability contours for the location of the gamma-ray source 3EG J1027-5817.

separating local clouds from Carina arm gas. According to their results it is possible to determine that toward $l \sim 284^{\circ}$ gas showing velocities around $\sim-15 \mathrm{~km} \mathrm{~s}^{-1}$ belongs to the Carina arm and is located at about $3 \mathrm{kpc}$, i.e., the distance of WR 21a. In Fig. 2 it can be appreciated that the gas distribution changes at about $v \sim-14$ to $-12 \mathrm{~km} \mathrm{~s}^{-1}$. We are going to focus on gas with $\Delta v=-20$ to $-12 \mathrm{~km} \mathrm{~s}^{-1}$ because, according to the $\mathrm{CO}$ results, its kinematical distance is compatible with that of the target star. The H I column density at the mentioned velocity interval is presented in Fig. 3. From the plot of Grabelsky et al. (1987), CO gas with velocities between -20 and $-10 \mathrm{~km} \mathrm{~s}^{-1}$ is located between 2 and $\sim 3.5 \mathrm{kpc}$. This fact helps to constrain an approximate error in the H I distance of $\leq 1 \mathrm{kpc}$. At larger velocities, Grabelsky et al. claimed that gas related to RCW 49 shows velocities of $-5 \mathrm{~km} \mathrm{~s}^{-1}$, and placed it at $4 \mathrm{kpc}$. A distance of $\sim 5 \mathrm{kpc}$ can be derived, for Carina gas with velocities near $0 \mathrm{~km} \mathrm{~s}^{-1}$. If the $\mathrm{H}$ I follows the motions proposed by Grabelsky et al., it is reasonable to suggest that gas with a velocity of $-14 \mathrm{~km} \mathrm{~s}^{-1}$ lies at $3 \mathrm{kpc}$.

Figure 3 shows the presence of gas at a distance compatible with that of the stellar system. Due to the strong continuum source RCW 49, part of the HI gas is not emitting but absorbing. At a distance of $3 \mathrm{kpc}$, the visible neutral gas coincident with the position of the EGRET source would sum up about $1500 M_{\odot}$, which can be considered as a lower limit for the masses of clouds at the distance we are interested on.

By means of Antenna I at IAR $\left(H P B W=12.4^{\circ}\right)$ we also measured the $\mathrm{H} 125 \alpha(3326.9880 \mathrm{MHz})$ radio recombination 


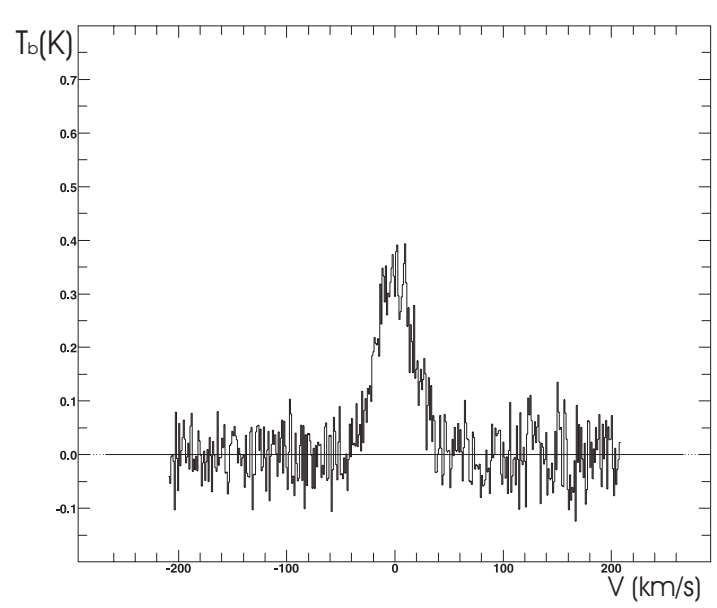

Fig. 4. $3.3 \mathrm{GHz}$ radio recombination line $\mathrm{H} 125 \alpha$ towards RCW 49 . $H P B W=12.4^{\circ}$.

line $(\operatorname{RRL})$ towards $(l, b)=\left(284.32^{\circ},-0.34^{\circ}\right)$. Since RRLs are typically produced by $\mathrm{H}$ II regions, the detected emission (see Fig. 4) is likely to be mostly from RCW 49. We measure a center velocity of $v=+0.6 \pm 1.4 \mathrm{~km} \mathrm{~s}^{-1}$, similar to those found at other RRLs in RCW 49 (e.g. Caswell \& Haynes 1987).

The kinetic energy needed to form a typical bubble around WR 21a can be computed as $E_{\mathrm{k}}=0.5 M_{\mathrm{sh}} v_{\mathrm{exp}}^{2}=10^{49} \mathrm{erg}$ if we assume a shell mass of $M_{\mathrm{sh}}=10000 M_{\odot}$ and an expansion velocity of $v_{\exp }=10 \mathrm{~km} \mathrm{~s}^{-1}$, which are typical parameters of neutral shells detected around massive Of stars (Cappa \& Benaglia 1998; Benaglia \& Cappa 1999).

The stellar wind luminosity can be expressed as $L_{\mathrm{w}}=$ $0.5 \dot{M} v_{\infty}^{2}$. Using the values of $\dot{M}$ and $v_{\infty}$ given in Table 1 , we find $L_{\mathrm{w}, \mathrm{WR}}=3.8 \times 10^{37} \mathrm{erg} \mathrm{s}^{-1}$, and $L_{\mathrm{w}, \mathrm{O}}=4.4 \times 10^{37} \mathrm{erg} \mathrm{s}^{-1}$.

Finally, a wind mechanical energy $E_{\mathrm{w}}=L_{\mathrm{w}} t=2.6 \times$ $10^{51}$ erg per Myr is obtained if both stars are considered, and $E_{\mathrm{w}}=1.2 \times 10^{51} \mathrm{erg}$ per Myr for only WN6. It can be seen that the energy deposited by the wind is much larger than the energy needed to create a typical bubble.

\section{The X-ray history of WR 21a}

Among the Wolf-Rayet stars, WR 21a is especially prominent in X-rays: despite its modest optical magnitude, it is one of the five or six brightest sources in both apparent and absolute terms. Since the discovery of 1E 1024.0-5732 in 1979 with the Einstein Observatory by Goldwurm et al. (1987), through its identification with a Wolf-Rayet star by Mereghetti et al. (1994), WR 21a has been observed on ten separate occasions in X-rays as shown in Table 3. All of these data are available through the HEASARC. As we mentioned before, Reig (1999) compared his 1997-RXTE data with earlier Einstein-IPC and ROSAT-PSPC measurements to show that the X-ray luminosity had apparently been steadily rising by about a factor of five in the eighteen years since its discovery and argued that this showed WR 21a is a long-period CWB of the type exemplified by WR 140 (Williams et al. 1990).

Though in general terms the latter is probably correct, the details are more complicated. First, the X-ray model which Reig used had too high an absorbing column density

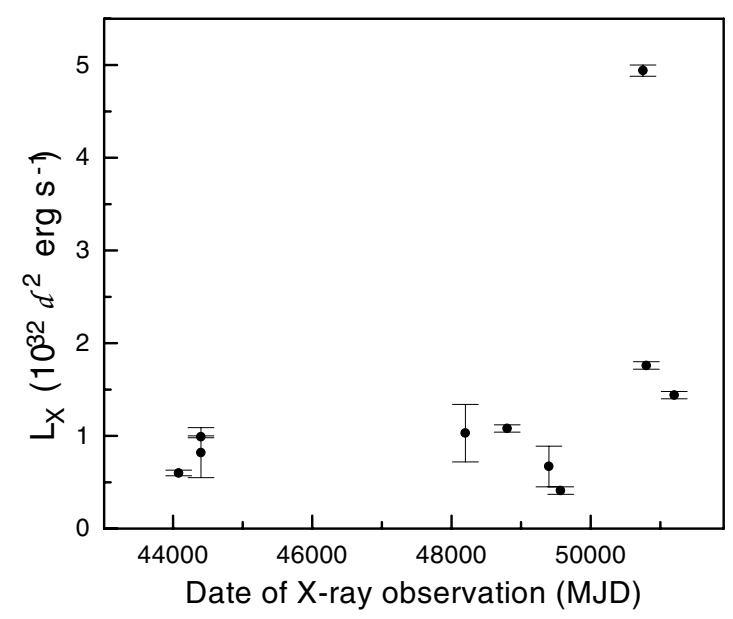

Fig. 5. The history of WR 21a's $1-2 \mathrm{keV}$ X-ray luminosity since its discovery in 1979 with the Einstein Observatory, through measurements in the early 1990s with ROSAT to the RXTE and ASCA measurements of 1997 and 1998. WR 21a is bright and the luminosity errors are usually smaller than the plotting symbols. The luminosities were calculated assuming the spectrum did not change shape and the range 1-2 keV was chosen to provide good overlap between instruments whose sensitivity ranges were different. The highest point is from $R X T E$ which, as explained in the text, probably included a significant contribution from nearby unresolved point-source and diffuse emission.

$\left(N_{\mathrm{H}}=2.9 \times 10^{22} \mathrm{~cm}^{-2}\right)$ to be consistent with the the soft $\mathrm{X}$-ray data; and second, the faintness of the pair of ROSAT-HRI measurements made in 1994 (Belloni \& Mereghetti 1994) contradicts the apparent inexorable rise in luminosity since 1979. Since the RXTE observation, two archived sets of ASCA data have also become available that are useful for bridging the soft X-ray images and the hard X-ray collimator data. The first ASCA observation took place two months after Reig's RXTE pointing though WR 21a was not the main objective and thus appeared near the edge of the GIS field-of-view, one of the two ASCA instruments that provided imaging X-ray spectroscopy. As a consequence there are no data from the SIS, the other instrument. On the other hand, data are available from the full set of ASCA instruments from an observation performed nearly a year later at the end of 1998.

We have obtained and analysed with XSPEC v11.2 all the archived spectra and associated response matrices available from the HEASARC. Despite the obvious changes in overall luminosity, we could find no evidence of any changes in the shape of the spectrum which, within the limited energy resolution available, seems to be consistent with the relatively hot few keV thermal plasma observed from the Wolf-Rayet binaries exemplified by WR 140 (Pollock et al. 2005), in contrast with the cooler temperatures more typical of the intrinsic emission of single stars (see for example the studies on the presumably single WN stars WR 1, Ignace et al. 2003; WR 6, Skinner et al. 2002b; and WR 110, Skinner et al. 2002a). The spectrum was modeled as an absorbed Bremsstrahlung continuum with additional emission lines of $\mathrm{Si}, \mathrm{Mg}$ and $\mathrm{Ne}$. The best-fit values of column density and temperature were $N_{\mathrm{H}}=7.0 \pm 0.6 \times 10^{21} \mathrm{~cm}^{-2}$, about a factor of 4 lower than 
Table 3. X-ray observations of WR 21a.

\begin{tabular}{lllrcc}
\hline \hline Date & Dataset ID & Instrument & $\begin{array}{r}\text { Time } \\
(\mathrm{s})\end{array}$ & $\begin{array}{c}\text { Count rate } \\
\left(\mathrm{s}^{-1}\right)\end{array}$ & $\begin{array}{c}L_{X}(1-2 \mathrm{keV}) \\
\left(10^{32} \mathrm{~d}_{\mathrm{kpc}}^{2} \mathrm{erg} \mathrm{s}^{-1}\right)\end{array}$ \\
\hline $1979-07-13$ & 3341 & Einstein-IPC & 1324 & $0.036 \pm 0.002$ & $0.60 \pm 0.03$ \\
$1980-07-08$ & 7715 & Einstein-IPC & 7680 & $0.0592 \pm 0.0002$ & $0.99 \pm 0.01$ \\
$1980-07-10$ & 7716 & Einstein-HRI & 7006 & $0.006 \pm 0.002$ & $0.82 \pm 0.27$ \\
$1990-10-01$ & RS932717N00 & RASS & 180 & $0.0778 \pm 0.0233$ & $1.03 \pm 0.31$ \\
$1992-07-29$ & RP400329N00 & ROSAT-PSPC & 8396 & $0.0817 \pm 0.0031$ & $1.08 \pm 0.04$ \\
$1994-01-14$ & RH201611N00 & ROSAT-HRI & 696 & $0.0177 \pm 0.0059$ & $0.67 \pm 0.22$ \\
$1994-07-31$ & RH201611A01 & ROSAT-HRI & 17906 & $0.0108 \pm 0.0012$ & $0.41 \pm 0.04$ \\
$1997-11-29$ & 301120101 & RXTE-PCA & 12000 & $7.088 \pm 0.079$ & $4.94 \pm 0.06$ \\
$1998-01-21$ & 26014000 & ASCA-GIS2 & 36858 & $0.0518 \pm 0.0012$ & $1.76 \pm 0.04$ \\
$1998-12-27$ & 46009000 & ASCA-GIS2 & 21174 & $0.0817 \pm 0.0020$ & $1.44 \pm 0.04$ \\
& & ASCA-SIS0 & 21788 & $0.1208 \pm 0.0024$ & \\
\hline
\end{tabular}

Reig's (1999) value, and $k T=3.3 \pm 0.2 \mathrm{keV}$. This empirical approach has the natural advantage of reproducing the range of ionization species in the spectrum, notably the simultaneous presence of the lines of SiXIII and SiXIV. The alternative fits given by XSPEC's plasma models were slightly worse but gave completely consistent best-fit values of column density and temperature.

The luminosities reported in Table 3 are for a joint fit to all the available spectra with only the luminosities free to vary between observations. The resulting lightcurve is shown in Fig. 5. The X-ray variability is apparently irregular though the measurements are spaced at such large intervals with respect to the newly-discovered period of weeks (Niemela et al. 2005) that it will only be possible to tell if it is related to the binary orbit once a precise orbit is available. Some care is also required with the brightest point that came from RXTE, the only instrument here with no imaging capabilities. Though Reig made a correction for the emission from other nearby sources by adding an extra spectral component with fixed parameters, this is quite uncertain, because of the extensive diffuse emission and the strength of the point sources enumerated by Belloni \& Mereghetti (1994), of which 1E 1022.2-5730 is of similar spectral shape to WR 21a.

\section{Connection with the EGRET source?}

The nature of the EGRET unidentified gamma-ray sources (Hartman et al. 1999) has become one of the most intriguing questions in astrophysics (e.g. Romero 2001). In the particular case of 3EG J1027-5817, the nearby X-ray source associated with WR 21a is indicated in the Third EGRET catalog as a potential counterpart.

The presence of a non-thermal contribution to the radio spectrum of WR 21a implies the existence of a population of relativistic particles in the source, which are probably accelerated at the CWR by diffusive shock acceleration (e.g. Bell 1978a,b) and cool by synchrotron emission in the local magnetic field. Electrons can also cool in such an environment through inverse-Compton interactions with stellar UV photons, producing non-thermal X-rays and gamma-rays (e.g. Pollock 1987; Benaglia \& Romero 2003). However, the facts that the X-ray emission from the system can be correctly modeled as thermal Bremsstrahlung and that the source has not been directly detected at gamma-rays suggest that the magnetic energy density in the CWR should largely exceed the photon energy density, implying significantly shorter cooling timescales for the synchrotron mechanism.

The same mechanism that accelerates the electrons should also operate on the ions. Synchrotron losses are not relevant for protons in the environment of the CWR. The maximum energy they can achieve will be determined by the photo-pion losses in the UV stellar field and by the size constraint imposed by the limited space available for the acceleration process. In the present case, where the CWR is not resolved and the geometry of the system remains unknown, we cannot calculate the highenergy cutoff for the non-thermal proton distribution. A value between 10 and $100 \mathrm{GeV}$ seems not unreasonable (see Benaglia \& Romero 2003).

It could be the case that some of these protons diffuse up to a nearby cloud where they might be trapped in the magnetic field, which is expected to be higher than the average value in the ISM (Crutcher 1999). Then they will interact there with the local material producing gamma-rays through $p+p \rightarrow p+p+\pi^{0}$ interactions and the subsequent $\pi^{0} \rightarrow \gamma+\gamma$ decays. The situation of a passive cloud irradiated by cosmic rays from some nearby accelerator has been discussed in detail by Black \& Fazio (1973) and by Aharonian \& Atoyan (1996). In the present case our ignorance on several basic parameters prevents accurate calculations, but there remains the possibility that a part of the flux detected from 3EG J1027-5817 could be originated in relativistic particles accelerated in the colliding wind region of WR 21a. Whether this is or not the case could be established through future observations of the gamma-ray source by instruments like AGILE and GLAST which could report the source position with higher accuracy.

\section{Summary and conclusions}

We have detected radio emission from WR 21 a at $4.8 \mathrm{GHz}$. The intensity of the source is $\sim 0.25 \mathrm{mJy}$. The non-detection at 
8.64 GHz implies a spectral index of $\alpha<0.3\left(S_{v} \propto v^{\alpha}\right)$, which significantly departs from a typical Bremsstrahlung spectrum. Combined thermal/non-thermal spectra are usually found in colliding-wind binaries. We suggest that this is also the case here, since the latest spectral determinations show that WR 21a is a system formed by WN6 and an early O companion (Reig 1999; Niemela et al. 2005). An upper limit for the system mass loss rate of $\dot{M}=4.8 \times 10^{-5} M_{\odot} \mathrm{yr}^{-1}$ is derived from the $4.8-\mathrm{GHz}$ radio flux density.

We have reanalyzed all X-ray observations of WR 21a in order to determine its time history in this waveband. Our results indicate, contrary to previous thought, that the X-ray flux has not been monotonically increasing since 1979 though the coverage is far too sparse to constrain the variability timescale. The $\mathrm{X}$-ray spectrum is consistent with a few $\mathrm{keV}$ thermal plasma, with no obvious non-thermal contribution.

Locally accelerated relativistic electrons in the CWR probably mainly cool by synchrotron emission at radio frequencies, with small inverse-Compton losses, of which there was no $\mathrm{X}$-ray evidence. If protons are also accelerated at the collidingwind shocks, then they might diffuse through the ISM up to nearby clouds, where they might interact with an enhanced $\mathrm{HI}$ density to produce gamma-rays from $\pi^{0}$-decays.

Future observations with both X-ray and gamma-ray instruments like CHANDRA, AGILE and GLAST can shed additional light on the nature of the high-energy emission in this interesting region. Detailed knowledge, on the other hand, of the orbital parameters of the system WR 21a will allow more sophisticated models to be built of the radiative processes taking place in the colliding-wind region.

Acknowledgements. We thank Virpi Niemela for discussions on this source. This research has been supported by the Argentine agency ANPCyT through grant PICT 03-13291.

\section{References}

Aharonian, F. A., \& Atoyan, A. M. 1996, A\&A, 917, 928

Bell, A. R. 1978a, MNRAS, 182, 147

Bell, A. R. 1978b, MNRAS, 182, 443

Belloni, T., \& Mereghetti, S. 1994, A\&A, 286, 935

Benaglia, P., \& Cappa, C. E. 1999, A\&A, 346, 979

Benaglia, P., Romero, G. E., Stevens, I. R., \& Torres, D. F. 2001a, A\&A, 366, 605

Benaglia, P., Cappa, C. E., \& Koribalski, B. S. 2001b, A\&A, 372, 952

Benaglia, P., \& Romero, G. E. 2003, A\&A, 399, 1121

Benaglia, P., \& Koribalski, B. 2004, A\&A, 416, 171

Bignami, G. F., \& Hermsen, W. 1983, ARA\&A, 21, 67

Black, J. H., \& Fazio, G. G. 1973, ApJ, 185, L7

Brand, J., \& Blitz, L. 1993, A\&A, 275, 67

Cappa, C. E., \& Benaglia, P. 1998, AJ, 116, 1906

Caraveo, P. A. 1983, Space Sci. Rev., 36, 207

Caraveo, P. A., Bignami, G. F., \& Goldwurm, A. 1989, ApJ, 338, 338

Cassé, M., \& Paul, J. A. 1980, ApJ, 237, 236

Caswell, J. L., \& Haynes, R. F. 1987, A\&A, 171, 261

Chapman, J. M., Leitherer, C., \& Koribalski, B. S., et al. 1999, ApJ, 518,890

Churchwell, E., Whitney, B. A., Babler, B. L., et al. 2004, ApJS, 154, 322

Crutcher, R. M. 1999, ApJ, 520, 706
Dieters, S. W., Hill, K. M., \& Watson, R. D. 1990, IAU Inf. Bull., 3500

Dougherty, S. M., Pittard, J. M., Kasian, L., et al. 2003, A\&A, 409 217

Eichler, D., \& Usov, V. 1993, ApJ, 402, 271

Fich, M., Blitz, L., \& Stark 1989, A\&A, ApJ, 342, 272

Grabelsky, D. A., Cohen, R. S., Bronfman, L., et al. 1987, ApJ, 315, 122

Goldwurm, A., Caraveo, P. A., \& Bignami, G. F. 1987, ApJ, 322, 349

Goss, W. M., Radhakrishnan, V., Brooks, J. W., \& Murray, J. D. 1972, ApJS, 24, 123

Hamann, W.-R., Koesterke, L., \& Wessolowski, U. 1995, A\&A, 299, 151

Hartman, R. C., Bertsch, D. L., Bloom, S. D., et al. 1999, ApJS, 123, 79

Hertz, P., \& Grindlay, J. E. 1984, ApJ, 278, 137

Ignace, R., Oskinova, L. M., \& Brown, J. C. 2003, A\&A, 408, 353

Koribalski, B., Johnston, S., Weisberg, J. M., \& Wilson, W. 1995, MNRAS, 441, 752

van der Hucht, K. A. 2001, New Astron. Rev., 45, 135

Leitherer, C., Chapman, J. M., \& Koribalski, B. 1997, ApJ, 481, 898

Manchester, R. N., Robinson, B. J., \& Goss, W. M. 1970, Aust. J. Phys., 23, 751

McClure-Griffiths, N. M., Green, A. J., Dickey, J. M., et al. 2001, ApJ, 551,394

Mereghetti, S., Belloni, T., Shara, M., \& Drissen, L. 1994, ApJ, 424, 943

Moffat, A. F. J., Shara, M. M., \& Potter, M. 1991, AJ, 102, 642

Morras, R., \& Cappa, C. E. 1995, IAR Internal Report, No. 74

Mücke, A., \& Pohl, M. 2002, in Interacting Winds from Massive Stars, ed. A. F. J. Moffat, \& N. St-Louis, ASP Conf. Ser., 260, 355

Niemela, V., et al. 2005, in preparation

Nolan, P. L., Tompkins, W. F., Grenier, I. A., \& Michelson, P. F. 2003 , ApJ, 597, 615

Nugis, T., \& Lamers, H. J. G. L. M. 2000, A\&A, 360, 227

Reig, P. 1999, A\&A, 345, 576

Pollock, A. M. T. 1987, A\&A, 171, 135

Pollock, A. M. T., Corcoran, M. F., Stevens, I. R., \& Williams, P. M. 2005, ApJ, in press

Prinja, R. K., Barlow, M. J., \& Howarth, I. D. 1990, ApJ, 361, 607

Roberts, M. S. E., Romani, R. W., \& Kawai, N. 2001, ApJS, 133, 451

Rodgers, A. W., Campbell, C. T., \& Whiteoak, J. B. 1960, MNRAS, 121,103

Romero, G. E. 2001, in The Nature of Unidentified Galactic High-Energy Gamma-Ray Sources, ed. A. Carramiñana, O. Reimer, \& D. J. Thompson (Kluwer), 267, 67

Romero, G. E., Benaglia, P., \& Torres, D. F. 1999, A\&A, 348, 868

Skinner, S. L., Zhekov, S. A., Güdel, M., \& Schmutz, W. 2002a, ApJ, 572,477

Skinner, S. L., Zhekov, S. A., Güdel, M., \& Schmutz, W. 2002b, ApJ, 579,764

Stevens, I. R., Blondin, J. M., \& Pollock, A. M. T. 1992, ApJ, 386, 265

Torres, D. F., Romero, G. E., Combi, J. A., \& Benaglia, P. 2001, A\&A, 370,468

Vacca, W. D., Garmany, C. D., \& Schull, J. M. 1996, ApJ, 460, 914

Vink, J. S., de Koter, A., \& Lamers, H. J. G. L. M. 2000, A\&A, 362, 295

Völk, H. J., \& Forman, M. 1982, ApJ, 253, 188

Wackerling, L. R. 1969, Mem. RAS, 73, 153

White, R. L. 1985, ApJ, 289, 698

White, R. L., \& Chen, W. 1992, ApJ, 387, L81

Whiteoak, J. B. Z., \& Uchida, K. I. 1997, A\&A, 317, 563

Williams, P. M., van der Hucht, K. A., Pollock, A. M. T., et al. 1990, MNRAS, 243, 662 